\title{
ESTRATEGIAS TECNOLÓGICAS Y CONJUNTO LÍTICO DEL CONTEXTO PALEOINDIO DE CUEVA LAGO SOFÍA 1, ÚLTIMA ESPERANZA, MAGALLANES
}

\author{
DONALD JACKSON S. ${ }^{*}$ Y ALFREDO PRIETO I.*
}

\begin{abstract}
RESUMEN
Se presenta una descripción del conjunto lítico del sitio Paleoindio de Lago Sofía 1 y su relación con el contexto y asociaciones culturales. Se discuten las estrategias tecnológicas implicadas en relación a estos grupos Paleoindios con alta movilidad en un proceso de exploración inicial o incipiente colonización.

\section{TECHNOLOGICAL STRATEGIES AND LITHIC SET FROM THE PALEOINDIAN CONTEXT OF CUEVA DEL LAGO SOFÍA 1, ÚLTIMA ESPERANZA, MAGALLANES}

ABSTRACT

The paper presents a description of the lithic sets of the Paleoindian site of Lago Sofía 1 and its cultural
\end{abstract} \begin{abstract}
associations. We discuss the technological strategies of these highly mobile Paleoindian groups in the context of initial exploration or early colonization processes.

\section{INTRODUCCIÓN}

El área de Última Esperanza es conocida por los numerosos hallazgos de fauna actualmente extinta en varias localidades, como son Cueva del Milodón (Moreno 1899, Hauthal 1899, Nordenskjöld 1900, Emperaire y Laming 1954, Saxon 1976, Borrero et al., 1991), Alero Dos Herraduras (Saxon 1976, Borrero et al. 1991, Massone 1991), Cueva Lago Sofía 4 (Borrero et al., 1997) y Cueva La Ventana (Alberdi y Prieto 2000), en todas las cuales se ha evaluado la eventual presencia de asociaciones

culturales hacia el Pleistoceno Tardío, no obstante sólo dos sitios atestiguan ocupaciones humanas paleoindias, por una parte Cueva del Medio (Nami 1986, 1987, Nami y Menegaz 1991) y por otra, Cueva Lago Sofía 1 (Prieto 1991).

La cueva 1 del Lago Sofía, constituye uno de estos excepcionales sitios paleoindios, en donde se ha registrado varias especies de fauna extinta, asociadas en un claro contexto cultural. Las evidencias de intervención humana están demostradas por huesos de fauna extinta con huellas antrópicas (golpes de fuego, cortes y fracturas), algunos artefactos de

* Departamento de Antropología, Facultad de Ciencias Sociales, Universidad de Chile, Casilla 10115, Santiago.

E-mail: djackson@uchile.cl

** Centro de Estudios del Hombre Austral, Instituto de la Patagonia, Universidad de Magallanes y Centro de Estudios del Cuaternario de Fuego-Patagonia (CEQUA). Casilla 113-D, Punta Arenas. E-mail: aprieto@aoniken.fc.umag.cl
\end{abstract}


hueso, instrumental lítico y un fogón baciforme en contextos estratigráficos datado hacia finales del Pleistoceno.

El instrumental lítico, objeto del presente estudio, está constituido por escasos instrumentos formatizados y desechos de talla, cuyo análisis ha permitido reconocer algunas singularidades morfológicas y tecnológicas, formas de aprovechamiento de las materias primas, así como la identificación de la función genérica de algunos de los instrumentos formalizados y la determinación de lascas de filos vivos utilizadas como instrumentos expeditivos. También se hacen algunos alcances sobre la cadena operativa representada en el contexto.

Aun cuando la muestra recuperada de las excavaciones estratigráficas es pequeña, lo excepcional de estos conjuntos paleoindios, amerita su estudio exhaustivo, en la perspectiva de ir modelando las distintas estrategias tecnológicas empleadas por los primeros poblamientos del extremo austral del continente.

\section{EL SITIO Y SU CONTEXTO CULTURAL}

La Cueva Lago Sofía 1 (CLS 1) se encuentra localizada al noreste de Cueva del Milodón, situada al pie del cerro Benítez, en el seno de Última Esperanza. Su emplazamiento se sitúa inmediatamente al noreste del lago Sofía.

La cueva está a los pies del cerro Campana con formaciones rocosas de conglomerados con bandas de lutitas de la formación Cerro Toro. La erosión de las capas más blandas ha generado su formación, en forma similar a como ocurrió en Cueva del Milodón (Wellman 1972) y en otras cuevas y aleros de la localidad. Se trata de una cueva bien protegida, que se presenta como una oquedad oblicua respecto al plano circundante que corresponde a una extensa terraza de $150 \mathrm{msnm}$. Tiene una extensión de cerca de $30 \mathrm{~m}$ de largo por 8 de ancho y $5 \mathrm{~m}$ de altura máxima y se ha dividido en tres sectores: uno que va desde la entrada hasta una gran plancha de roca caída del techo en el sector central; otro hacia la parte posterior con un área libre de este derrumbe, y un tercero, en el fondo, en forma de una pequeña cámara con una ligera inclinación hacia arriba (Prieto 1991: 78).

Grandes planchones de conglomerados desprendidos desde el techo de la cueva "sellaron" una buena parte de la superficie ocupable de ella. Los depósitos estratigráficos muestran desde arriba hacia abajo una sucesión de estratos discretos (1a, b y c) con abundantes intrusiones de carbonato de calcio formando potentes lentes blanquizcos en los primeros $40 \mathrm{~cm}$, luego tres estratos subyacentes (2a, $2 \mathrm{~b}$ y 3 ) de colores moreno, amarillento y marrón respectivamente. Estos estratos son más delgados que los anteriores y se encuentran en casi todas las cuadrículas, excepto en la cámara. Los estratos 2 y 3 se encuentran "sellados" por los bloques desprendidos desde el techo en el sector central y delantero de la cueva (Prieto 1991).

La cueva presenta dos ocupaciones, una de ellas del período IV situada en el sector de la cámara, correspondiente a una cremación de tres individuos, dos adultos y un niño. Este episodio fue dispuesto sobre los escasos sedimentos depositados en la cámara y pudieron remover algunos huesos de fauna extinta previamente depositados. Este evento ocupacional fue datado entre los $3.950 \pm$ 60 y $3.915 \pm 60$ años AP (Ibíd).

El segundo y más antiguo evento ocupacional corresponde al período I asignable a grupos paleoindios. Se ubica en gran parte de la cueva bajo los bloques de conglomerados desprendidos desde el techo, es decir hacia el sector central de la cueva. En el estrato moreno (2a) se asentaron los grupos paleoindios. Un fogón baciforme excavado en el estrato amarillo casi al contacto con el marrón, desde la superficie de ocupación (estrato moreno) dio un fechado sobre carbón de $11.570 \pm$ 60 años AP (Prieto 1991), no obstante adicionales dataciones precisan la cronología de la ocupación entre los $10.710 \pm 70$ y los $10.140 \pm 120$ años AP (Massone y Prieto 2004).

Las evidencias culturales de este evento ocupacional, están constituidas por el fogón baciforme asociado a carbones y huesos con golpes de fuego, instrumentos líticos, un punzón elaborado en hueso largo de cánido así como retocadores extremo laterales (Jackson 1990) y restos de fauna extinta que incluye Equidae (Hippidion saldiasi), y Mylodontidae (Mylodon darwin), Canidae (Dusicyon avus), Ctenomyidae (Megamys sp.) y fauna actual que incluye Camelidae (Lama guanicoe). Algunos de estos huesos presentan fracturas intencionales, golpes de fuego y huellas de faenamiento, los que atestiguan una clara actividad humana junto con 
TABLA 1

\begin{tabular}{|l|c|c|c|}
\hline \multicolumn{1}{|c|}{ Categorías Identificadas } & Período I & Período IV & Totales \\
\hline Núcleos y Fragmento Guijarros & 2 & 0 & 2 \\
\hline Derivados de Núcleos y desechos & 29 & 107 & 136 \\
\hline Lascas y láminas retocadas & 2 & 4 & 6 \\
\hline Cuchillos & 1 & 0 & 1 \\
\hline Raederas & 3 & 6 & 9 \\
\hline Totales & 37 & 117 & 154 \\
\hline
\end{tabular}

las asociaciones estratigráficas al fogón y a los instrumentos líticos y de hueso (Prieto 1991, Alberdi y Prieto 2000).

Bajo la ocupación paleoindia, se registran restos de huesos de milodón los que han sido fechados en $12.990 \pm 490$ años AP, sin presentar evidencias culturales (Prieto 1991).

\section{LOS INSTRUMENTOS LÍTICOS Y SUS DESECHOS}

El total del conjunto lítico recuperado en las excavaciones del sitio $\left(32 \mathrm{~m}^{2}\right)$ incluye un total de 154 piezas (Tabla 1) de las cuales sólo 37 de ellas pudieron asignarse con seguridad a la ocupación paleoindia del Período I. Esta asignación se basó en la seguridad de su posición estratigráfica y asociaciones, en consideración que, por una parte, el colapso del bloque de conglomerado desprendido desde el techo, perturbó en algunos sectores la estratigrafía, y por otra, la cremación del Período IV, provocó también alteraciones en la estratigrafía y en consecuencia en la posición "absoluta" de algunos artefactos. En este sentido, es posible que algunas piezas asignadas al Período IV correspondan realmente a la ocupación paleoindia.

Para el nivel paleoindio, entre las escasas evidencias líticas, se registró un núcleo tendiente a poliédrico (96.6-68.7-45.4 mm) desde donde se obtuvieron lascas a partir de utilizar como plataforma de percusión, los planos formados por lascas previamente desprendidas. La presencia de un retoque marginal sobre uno de los bordes o arista del núcleo, sugiere su re-utilización como cepillo de carácter expeditivo. También se registró un fragmento de un pequeño guijarro fracturado (50-26-24 mm) longitudinalmente como parte de un núcleo (Fig. 1; 1 y 2).
Los derivados de núcleo fueron examinados bajo una lupa binocular (60x) para evaluar la eventual presencia de micro-huellas de uso (Semenov 1982, Lewenstein 1990). Sólo se detectaron dos lascas con indicios de utilización de sus filos vivos. En ambos casos se trata de lascas con dos bordes paralelos utilizados, con presencia de claro microastillamiento marginal simple, producto de su uso; una con ambos bordes convexos en ángulo de $54^{\circ}$ y $30^{\circ}$ que sugiere la acción de raer y raspar. La otra pieza presenta un borde ligeramente cóncavo en ángulo de $26^{\circ}$ y el borde opuesto ligeramente recto en ángulo de $29^{\circ}$ que probablemente se utilizaron para cortar y raspar (Fig. 1; 5 y 6).
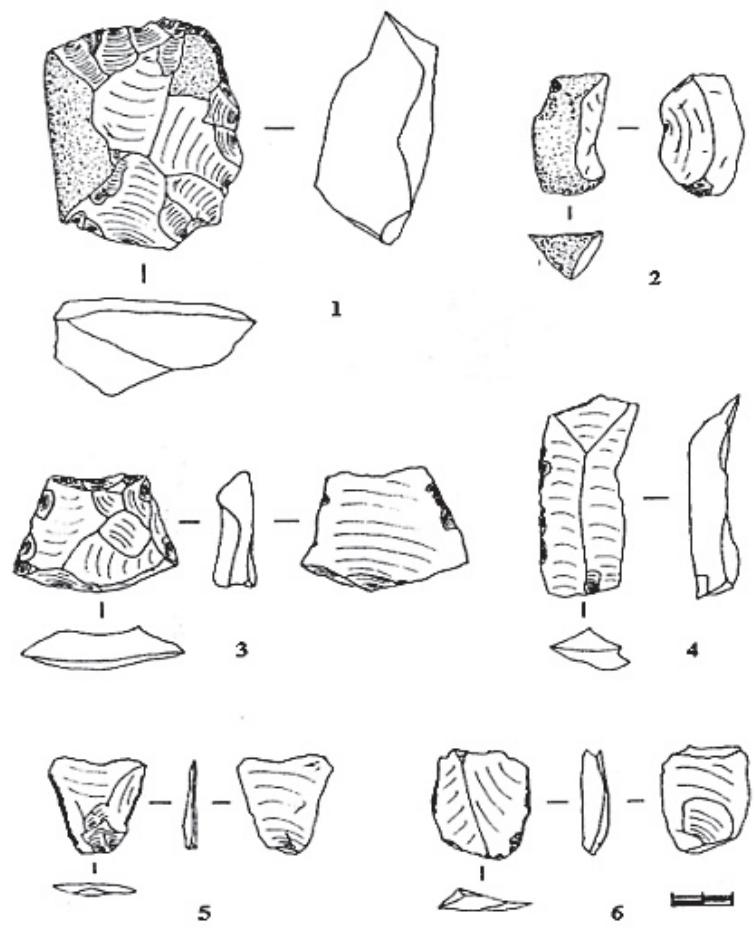

Fig. 1 
TABLA 2.

\begin{tabular}{|l|c|c|c|c|c|}
\hline & Media & Mediana & Moda & Std & Medidas Máximo/Mínimo en mm. \\
\hline Largo & 1.30 & 1.14 & $/$ & 0.77 & $2.97-0.57$ \\
\hline Ancho & 1.17 & 0.98 & 0.81 & 0.46 & $2.22-0.62$ \\
\hline Espesor & 0.32 & 0.26 & 0.21 & 0.21 & $0.91-0.11$ \\
\hline
\end{tabular}

Los derivados de núcleo y otros desechos incluyen un total de sólo 29 piezas de las cuales $19(65.5 \%)$ corresponden propiamente a lascas desprendidas desde el núcleo, en consideración de su tamaño, la presencia de corteza en el anverso y el registro de talones naturales y planos. Las restantes $10(34.5 \%)$ piezas incluyen desechos menores de talla y retoque con talones planos reducidos 0 rebajados. Menos de la mitad de las lascas (42.1\%) presenta corteza sobre su anverso. La relación largo vs. ancho de los derivados y desechos tiende a ser ligeramente similares (Ver Tabla 2).

Por otra parte, los instrumentos formalizados incluyen un excepcional cuchillo bifacial tendiente a lanceolado ancho de bordes convergentes en extremo
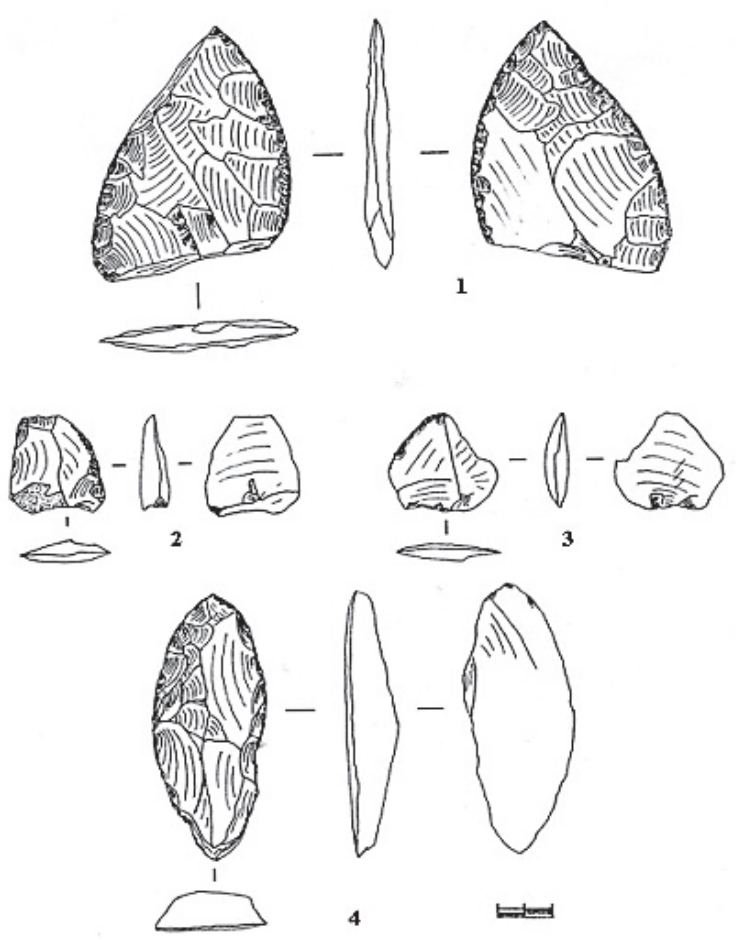

Fig. 2 agudo, bordes convexos en ángulos de $40^{\circ}$ y $47^{\circ}$ respectivamente y sección biconvexa. El astillamiento es bifacial y presenta un retoque ultramarginal en todos sus bordes de uso (Fig. 2; 1).

Otra de las categorías identificadas incluye tres raederas; dos laterales simples de borde activo convexo con ángulo de $60^{\circ}$ y $52^{\circ}$, con retoque ultramarginal, elaboradas en lascas conservando la cara de fractura como superficie de deslizamiento del instrumento, y una raedera doble apuntada de bordes activos convexos con retoque ultramarginal, en este caso agotada, utilizando la cara de fractura como superficie de deslizamiento. En dos de las piezas el eje tecnológico de la matriz (lascas y láminas) coincide con el eje morfo-funcional del instrumento (Fig. 2; 2, 3 y 4).

También se registró una lámina con retoque marginal simple discontinuo sobre un borde ligeramente recto en ángulo de $67^{\circ}$, probablemente producto del reavivado de un filo vivo desgastado (¿raer o cortar?), y una lasca con retoque marginal simple presumiblemente utilizada como cepillo. Las escasas modificaciones de ambos instrumentos sugieren un carácter expeditivo (Fig. 1; 3 y 4).

Las materias primas empleadas en la elaboración de estos instrumentos, así como en los desechos incluyen únicamente lutitas $(70 \%)$ y tobas (30\%), ambas materias primas locales y abundantes en los alrededores inmediatos del sitio.

\section{DISCUSIÓN Y CONCLUSIONES}

El contexto arqueológico de Lago Sofía 1 atestigua una ocupación paleoindia con claras asociaciones culturales y fauna extinta, datada hacia los 10.140 - 10.710 años AP. La presencia de un fogón baciforme, instrumentos líticos, de clara factura humana, un punzón y retocadores extremo-laterales (Jackson 1990), asociados a varias especies de fauna extinta en estratigrafía, aseguran una efímera ocupación de grupos Paleoindios hacia finales del Pleistoceno (Prieto 1991, Massone y Prieto 2004). 
El conjunto lítico recuperado en este contexto, corresponde a un pequeño número de instrumentos, núcleos y desechos que se encuentran en estratigrafía, claramente asociados a los restos de fauna extinta y en las proximidades del fogón "baciforme". Un número mayor de instrumentos y desechos presentes en el contexto, se excluyeron por no presentar asociaciones seguras con la ocupación Paleoindia, y aunque en su mayoría son atribuidos al Período IV, en un segundo momento ocupacional del sitio, es posible que algunas de estas piezas correspondan efectivamente al evento ocupacional Paleoindio.

Las escasas evidencias líticas registradas indican la presencia de instrumentos vinculados con las actividades de destazamiento y esencialmente del carneo de partes de presas trasladadas al sitio, donde debieron ser utilizadas las raederas, cuchillos y algunas lascas de filos vivos. Sólo una lasca retocada, así como el núcleo reutilizado como tajador, pudieron presumiblemente ser utilizados para labores de desbastado y cepillado de madera. En este sentido, hay que considerar que para el momento de ocupación en la zona sólo existían pequeños bosquetes en los valles recientemente deglaciados, los que debieron ser atractivos para la búsqueda de maderas para la confección de astiles y otros artefactos (Prieto 1991).

El núcleo y los escasos derivados de núcleo correspondiente a pequeñas lascas y desechos de retoque, sugieren una efímera actividad de procesamiento de instrumentos y reactivado de filos desgastados, registrándose una mínima parte de la cadena operativa implicada por la elaboración de los instrumentos identificados en el contexto.

Por otra parte, la formatización de los instrumentos líticos, como en el caso de las raederas y cuchillos, apunta a una estrategia tecnológica conservada donde el diseño de los instrumentos (Binford 1979, Nelson 1991) se orienta a una eficiencia funcional (confiabilidad) que supone su transporte y conservación en cazadores-recolectores de alta movilidad. Esto es coherente con el contexto estudiado, pues se trata de grupos paleoindios de alta movilidad.

Una estrategia de este tipo, también es consistente con modelos acerca de los procesos de exploración inicial (y colonización) de ambientes desconocidos previamente (Borrero 1994-95). En este sentido, la escasa variedad de materias primas utilizadas, únicamente lutitas y tobas, parece ser una función del conocimiento de la disponibilidad y accesibilidad de las materias primas líticas. Esto se confirma y contrasta con el conjunto lítico atribuido al Período IV, con una mayor variedad de materias primas, donde se registra además de lutitas y tobas, sílice amorfo y obsidiana, atestiguando un mayor conocimiento del entorno.

Por otra parte, el uso de materias primas locales de alta disponibilidad condujo a un escaso reactivado y al descarte de instrumentos aún con vida útil remanente. Si eventualmente existieron instrumentos en materias primas no locales de buena calidad, éstos no fueron descartados en el sitio, sino conservados y transportados.

El panorama descrito es similar al registrado en el sitio paleoindio de Cueva del Medio, a excepción de la presencia de piezas bifaciales (Nami 1986, 1987). El relativo sincronismo cronológico y cercanía espacial con este sitio, sugieren además de una misma modalidad cultural (Fell 1), alguna conexión a nivel del patrón de asentamiento en la exploración inicial o incipiente colonización de Última Esperanza, en los límites occidentales de lo que era habitable hacia finales del Pleistoceno.

\section{BIBLIOGRAFÍA}

ALBERDI, M. y A. PRIETO. 2000. Hippidion (Mammalia, Perissodactyla) de las cuevas de las provincias de Magallanes y Tierra del Fuego. Anales del Instituto de la Patagonia, Serie Ciencias Humanas (Chile) 28:147-171, Punta Arenas.

BINFORD, L. 1979. Organization and formation processes: looking al curated technologies. Journal of Anthropological Research 35:255-273.

BORRERO, L. 1994-95. Arqueología de Patagonia. Palimpsesto, Revista de Arqueología 4:9-69.

BORRERO, L.; J. L. LANATA y P. CÁRDENAS. 1991. Reestudiando cuevas: nuevas excavaciones en Última Esperanza, Magallanes. Anales del Instituto de la Patagonia, Serie Ciencias Humanas (Chile) 20:101110, Punta Arenas.

BORRERO, L.; F. MARTIN y A. PRIETO. 1997. La Cueva Lago Sofía 4, Última Esperanza: una madriguera del felino del Pleistoceno Tardío. Anales del Instituto de la Patagonia, Serie Ciencias Humanas (Chile) 25:103122, Punta Arenas.

EMPERAIRE, J. y A. LAMING. 1954. La grotte du Mylodon (Patagonie Occidentale). Journal de la Société D. Americanistes, XLIII: 173-205, Paris.

HAUTHAL, R. 1899. Reseña de los hallazgos en las cavernas de Última Esperanza. Revista del Museo de La Plata IX: 409-420. La Plata. 
JACKSON, DONALD. 1990. Retocadores extremo-laterales en contextos Paleo-Indios. Anales del Instituto de la Patagonia, Serie Ciencias Humanas (Chile) 19:121-124, Punta Arenas.

LEWENSTEIN, S. 1990. La función de los artefactos líticos por medio del análisis de huellas de uso. En: Nuevos Enfoques en el estudio de la Lítica (Edit. Arechavaleta), Instituto de Investigaciones Antropológicas, Universidad Autónoma de México.

MASSONE, MAURICIO. 1991. Estudio de la ceniza volcánica y sus implicancias en la interpretación de algunos registros arqueológicos de Chile Austral. Anales del Instituto de la Patagonia, Serie Ciencias Humanas (Chile) 20:111115, Punta Arenas.

MASSONE, M. y A. PRIETO. 2004. Evaluación de la modalidad Fell 1 en Magallanes. Chungará, Revista Chilena de Antropología, Volumen Especial, pp.303-315, Arica.

MORENO, FRANCISCO. 1899. Note on the discovery of the Miolania and of glossotherium (Neomylodon) in Patagonia. Nature, 60:396-398.

NAMI, HUGO. 1986. Excavación arqueológica y hallazgo de una punta de proyectil FELL I en la Cueva del Medio, Seno de Última Esperanza, Chile. Anales del Instituto de la Patagonia, Serie Ciencias Humanas (Chile) 16:103-109, Punta Arenas.

NAMI, HUGO. 1987. Cueva del Medio: perspectivas arqueológicas para la Patagonia Austral. Anales del Instituto de la Patagonia, Serie Ciencias Humanas (Chile) 17:73-106, Punta Arenas.

NAMI, H. y A. MENEGAZ. 1991. Cueva del Medio, aportes para el conocimiento de la diversidad faunística hacia el Pleistoceno Holoceno en Patagonia Austral. Anales del Instituto de la Patagonia, Serie Ciencias Humanas (Chile) 23:125-133, Punta Arenas.

NELSON, M. 1991. The Study of Technological Organization. En: Archaelogical Method and Theory, Vol.3, Editado por M. Schiffer, pp. 57-100. University of Arizona Press, Tucson.

NORDENSKJÖLD, E. 1900. Jackttagelser och fynd I Grottor vid Última Esperanza y sydvestra patagonien. Loglinga svenska vetenskaps-Akademien Handlingar, 33:1-24.

PRIETO, ALFREDO. 1991. Cazadores tempranos y tardíos en Cueva del Lago Sofía 1. Anales del Instituto de la Patagonia, Serie Ciencias Humanas (Chile) 20:75-99, Punta Arenas.

SAXON, EARL. 1976. La Prehistoria de Fuego-Patagonia: Colonización de un hábitat marginal. Anales del Instituto de la Patagonia, Serie Ciencias Humanas (Chile) 7:63-73, Punta Arenas.

SEMENOV, S. 1982. Tecnología Prehistórica. Editorial Akal, Madrid.

WELLMAN, R. 1972. Origen de la Cueva del Mylodón en Última Esperanza. Anales del Instituto de la Patagonia, Serie Ciencias Humanas (Chile) 3:97-101, Punta Arenas. 